\title{
Reversible posterior encephalopathy syndrome associated with late onset postpartum eclampsia: A case report
}

\author{
QI-YU BO ${ }^{1}$, XIU-HE ZHAO ${ }^{2}, \mathrm{XUE} \mathrm{YANG}^{2}$ and SHENG-JUN WANG ${ }^{2}$ \\ Departments of ${ }^{1}$ Operating Room and ${ }^{2}$ Neurology, Qilu Hospital, Shandong University, Jinan, Shandong 250012, P.R. China
}

Received June 20, 2015; Accepted June 23, 2016

DOI: $10.3892 /$ etm.2016.3533

\begin{abstract}
Late onset postpartum eclampsia (LPE) is defined by its onset at $>48 \mathrm{~h}$ after delivery. Reversible posterior encephalopathy syndrome (RPES) associated with LPE is uncommon, with the majority of RPES cases having a late postpartum onset within 4 weeks after childbirth. The present study reported the case of a 15 -year old female presenting with convulsions that began 5 weeks after delivery. A magnetic resonance imaging scan of the brain revealed multiple lesions in the cortex, subcortical region and deep white matter of the bilateral cerebellum, and occipital, frontal and parietal lobes. The clinical manifestations and radiological abnormalities were readily resolved subsequent to antihypertension and anticonvulsion treatment. In conclusion, the present rare case indicates that LPE should be considered as a potential diagnosis even at 4 weeks after delivery. Furthermore, clinicians should familiarize with the reversible radioimaging features of RPES, since early recognition and adequate treatment are important to the outcome of patients.
\end{abstract}

\section{Introduction}

Eclampsia refers to the occurrence of generalized convulsions in a woman during puerperium without an alternative identifiable cause. Approximately $50 \%$ of all cases of eclampsia occur after delivery, which is known as postpartum eclampsia (1). Late onset postpartum eclampsia (LPE) occurs $>48$ h after childbirth (2). Complications in eclampsia include acute live, renal failure and respiratory complications. Mortality in eclampsia is predominantly a result of intracerebral hemorrhage (2). Mortality rates could be lowered with antihypertensive therapy and anticonvulsants in patients with seizures (2). Reversible posterior encephalopathy syndrome (RPES), also termed reversible posterior leukoencephalopathy, refers to a clinical and radiological entity in which

Correspondence to: Dr Sheng-Jun Wang, Department of Neurology, Qilu Hospital, Shandong University, 107 Wen Hua Xi Road, Jinan, Shandong 250012, P.R. China

E-mail: junwang9999@sina.com

Key words: postpartum, eclampsia, late, reversible, encephalopathy patients often present with headaches, seizures and altered mental status, with reversible edematous changes in the cortex, subcortical region and deep white matter observed upon brain radioimaging (3). Eclampsia is one of the underlying clinical conditions predisposing patients to RPES (4). The majority of RPES cases with late postpartum onset present within 4 weeks after the delivery $(5,6)$. The present study reports a rare case of LPE-associated RPES, which occurred at 5 weeks after delivery in a 15-year-old female patient.

\section{Case report}

A 15-year-old female patient complained of headache and transient visual loss $\sim 5$ weeks after preterm delivery at 32 weeks of pregnancy. The patient had a fainting episode prior to admission to Qilu Hosptal of Shandong University (Jinan, China) in March 2012, with upward gaze deviation and tongue biting reported. The patient had experienced hypertension during the pregnancy without any headaches or convulsions, and presented mild leg edema, paroxysmal abdominal pain and nausea during the pregnancy and post partum. No previous major systemic disease and medicine use were reported. The patient was admitted to the emergency room, where she experienced two further generalized tonic-clonic seizures and became somnolent. Intravenous diazepine (10 mg) was administered immediately. The patient had a high blood pressure $(200 / 110 \mathrm{mmHg})$, which was successfully controlled by urapidil administration (100 $\mathrm{mg}$ per day), and presented a temperature of $38.3^{\circ} \mathrm{C}$.

A neurological examination indicated a somnolent state without any focal neurological signs. Laboratory blood tests revealed leukocytosis (white blood cell count, 16,000 cells $/ \mathrm{mm}^{3}$; normal range, 3,500-9,500 cells $/ \mathrm{mm}^{3}$ ) with left-shifting. A head magnetic resonance imaging (MRI) scan was performed, which indicated multiple foci of high signal intensity on T2-weighted images and fluid-attenuated inversion recovery (FLAIR) sequences in the cortical and subcortical white matter bilaterally, predominantly in the occipital, frontal and parietal lobes and cerebellum (Fig. 1). In addition, diffuse weighted imaging (DWI) revealed no evident abnormalities (Fig. 1). Following admission, the results of cardiovascular, respiratory, abdominal, neurological and ophthalmologic examinations were normal. Laboratory tests were also normal, including urinalysis, serum electrolytes, liver function, glucose and lactic acid levels, coagulation profiles, serological 
A

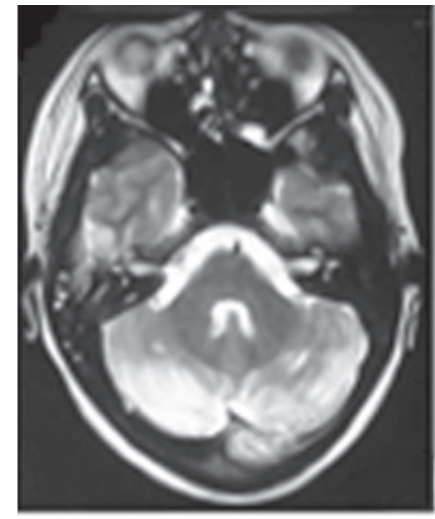

D

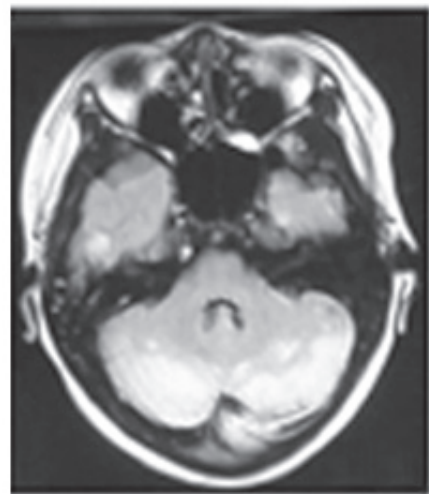

G

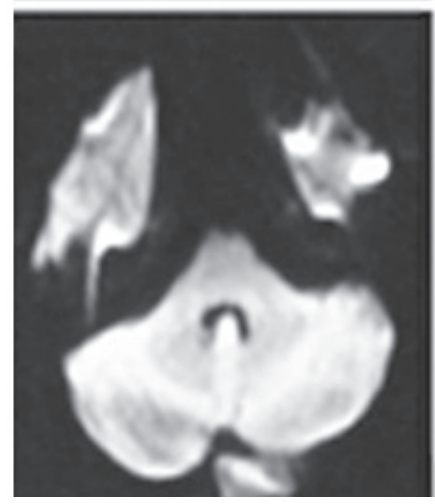

B

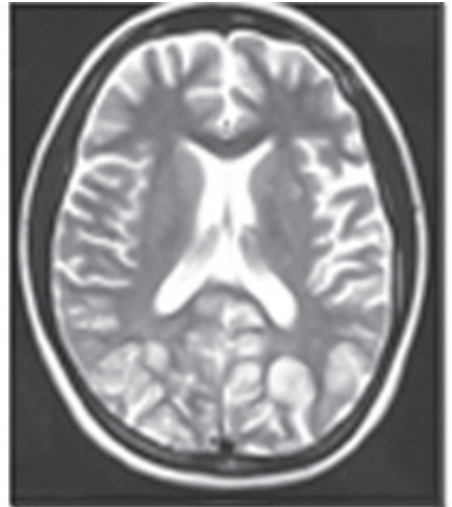

$\mathbf{E}$

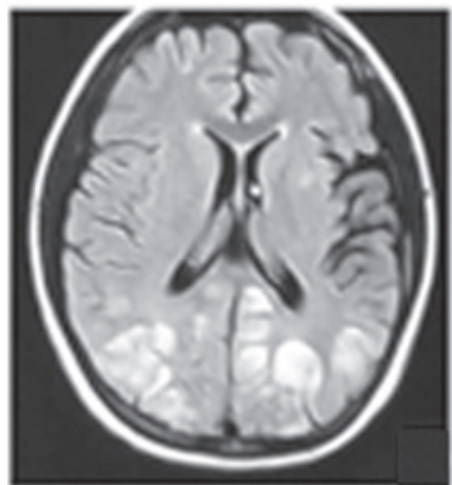

$\mathbf{H}$

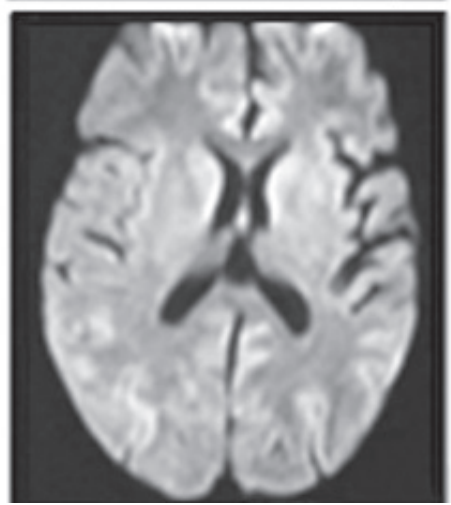

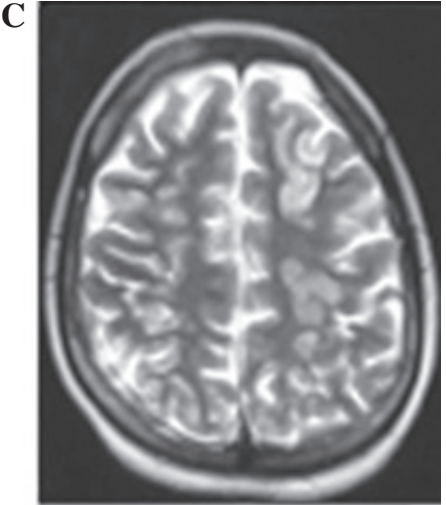

$\mathbf{F}$

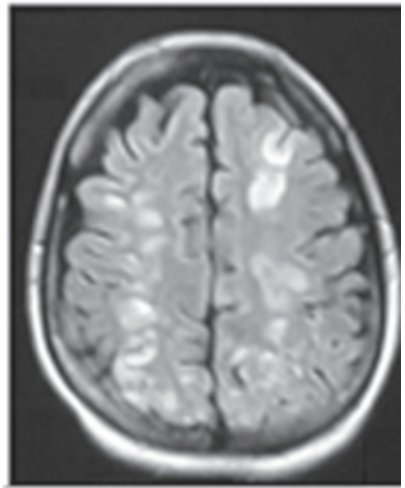

I

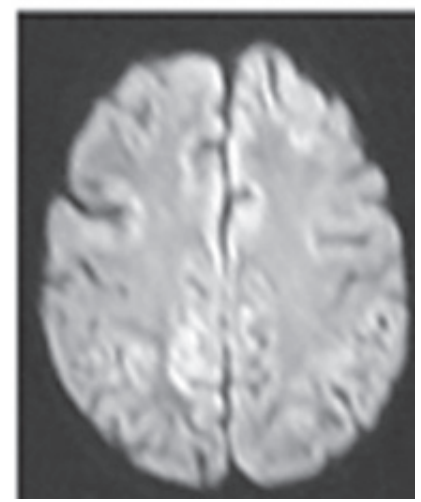

Figure 1. Magnetic resonance imaging scans of the head (prior to hospital admission). (A) Obvious high signal-intensity lesions in the deep white matter of the bilateral cerebellum on T2-weighted image (T2WI). (B) Multiple high signal-intensity lesions in the cortex and subcortical regions of occipital lobe on T2WI. (C) Multiple high signal-intensity lesions in the frontal-parietal watershed zone on T2WI. (D) High signal-intensity lesions in the deep white matter of the bilateral cerebellum on fluid attenuated inversion recovery (FLAIR) image. (E) Multiple high signal-intensity lesions in the occipital lobe on FLAIR image; (F) Multiple high signal-intensity lesions in frontal-parietal watershed zone on FLAIR image. (G) Mild high signal intensity lesions in the deep white matter of the bilateral cerebellum on the diffuse weighted image (DWI). (H) Mild high signal intensity lesions in the occipital lobe on DWI. (I) Mild high signal intensity lesions in the in frontal and parietal lobes on DWI.

assessment (including the venereal disease research laboratory test, and tests for thyroid hormones) and an autoimmune profile (which involved assessments for antinuclear antibodies, double strand DNA, antineutrophil cytoplasmic antibodies and antiphospholipid antibodies). A lumbar puncture was also performed, and the cerebrospinal fluid opening pressure, protein, glucose, immunoglobulin and the number of cells were found to be normal.

On day 4 after hospital admission, the patient received further head MRI and magnetic resonance arteriography/magnetic resonance venography (MRA/MRV) scans. The hyperintensity zones on T2-weighted images of the subcortex and white matter in the cerebellum, and occipital, frontal and parietal lobes were markedly decreased. The MRA scan revealed cerebral vascular focal vasodilation and vasoconstriction features with a string-of-beads appearance (Fig. 2), predominantly in the vessels of the posterior circulation of the brain. In addition, there was no intracranial sinus thrombosis; however, the MRV indicated venous sinus dilation (Fig. 2). Based on the results of tests, the diagnosis of reversible posterior encephalopathy induced by LPE was suspected. The patient was administered cefoperazone (4 mg per day), nimodipine (10-20 mg per day) and mannitol (250-500 $\mathrm{ml}$ per day). The temperature and blood pressure were normalized following treatment, and the patient experienced no further seizures during the remaining of the hospital stay. On day 11 after admission, the patient received further head MRI examination that indicated an almost complete resolution of the previous abnormalities (Fig. 3). Subsequently, the patient was discharged without any neurological sequela. 

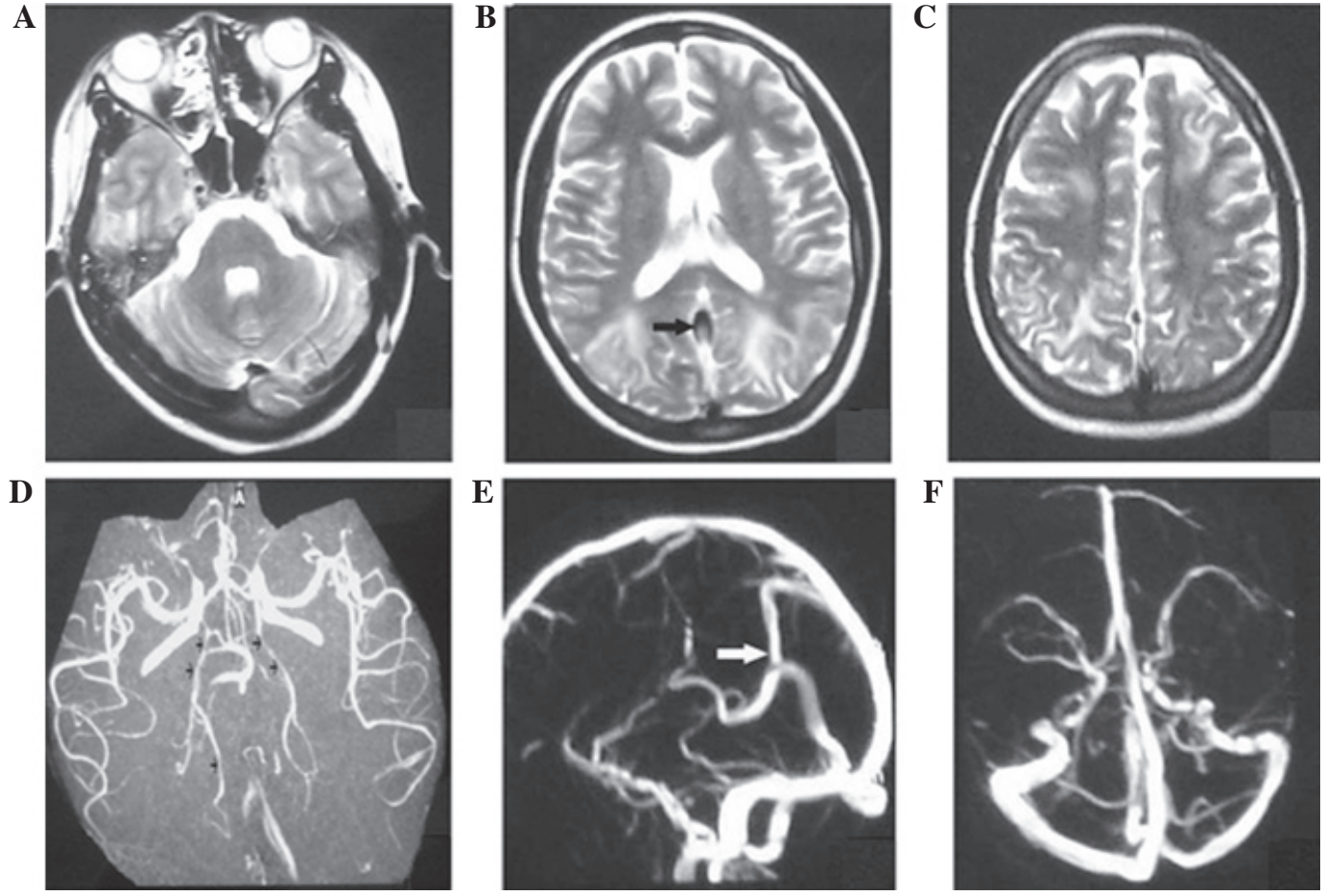

Figure 2. Magnetic resonance imaging scans of the head four days after hospital admission. (A) Moderately high signal-intensity lesions in the deep white matter of the bilateral cerebellum on T2WI. (B) Moderately high signal-intensity lesions in the cortex and subcortical regions of occipital lobe on T2WI. (C) Moderately high signal-intensity lesions in the frontal-parietal watershed zone on T2WI. (D) Focal vasodilation and vasoconstriction in addition to a string-of-beads appearance in the posterior cerebral arteries bilaterally (short arrows) on magnetic resonance arteriography (MRA) image. (E) The cerebral venous system shows vasodilation appearance (long arrow) on magnetic resonance venography (MRV) image. (F) No evidence of venous sinus thrombosis was found on MRV image.
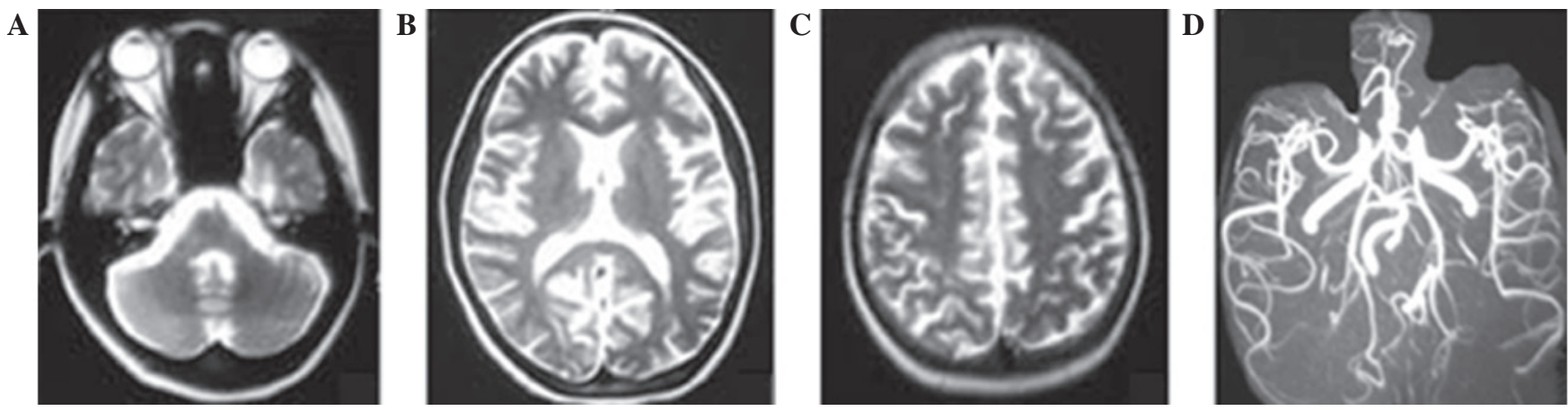

Figure 3. Magnetic resonance imaging scans of the head 11 days after hospital admission. (A) Slightly high signal-intensity lesions in the bilateral cerebellum on T2WI. (B) Slightly high signal-intensity lesions in the occipital lobe on T2WI. (C) Slight high signal-intensity lesions in the frontal-parietal watershed zone on T2WI. (D) Magnetic resonance arteriography images showed no evident features of vessel constriction and dilation.

The follow-up neurological examination after 1 month showed no abnormalities. During the two months follow-up examinations, no neurological symptoms were found.

\section{Discussion}

The diagnosis of RPES associated with LPE in the patient of the current study was based on the reversible clinical course, the increasing of blood pressure, the detection of vasogenic edema on a head MRI scan and the recent medical history of childbirth. The differential diagnosis for frequent convulsions in the puerperal period includes ischemic stroke, encephalitis, meningitis, mitochondrial encephalopathy, Hashimoto's encephalopathy, electrolyte or endocrine disturbances, vasculitis, inflammatory demyelinating diseases and neoplastic diseases (7). Laboratory assessments, cerebrospinal fluid examination, reversal of the head MRI features following treatment and good outcome without the use of immunosuppressive treatment excluded the aforementioned differential diagnoses. In addition, the patient only experienced a mild headache during the puerperal period, which excluded the possibility of reversible cerebral vasoconstriction syndrome, which is characterized by severe or thunderclap headaches and reversible constriction of cerebral arteries. Thus, the diagnosis of LPE-associated RPES was established in the current patient.

LPE is distinguished from early onset postpartum eclampsia by an onset that occurs $>48 \mathrm{~h}$ after childbirth (2). 
LPE may occur without any pre-eclamptic prodromes. The delayed onset and the atypical clinical presentation of LPE may lead to misdiagnosis. The patient reported in the present study did not demonstrate typical pre-eclamptic prodromes, although she experienced hypertension and limb edema during her pregnancy, and presented with convulsions 5 weeks after the delivery. To the best of our knowledge, only one patient reported in the literature experienced LPE with onset later than 4 weeks postpartum (8).

LPE may manifest as RPES in which the most frequently affected areas are the subcortical white matter in the cerebral posterior circulation region (9). The less frequently affected areas include the frontal lobe, temporal lobe and basal ganglia. The characteristic radioimaging findings of RPES are reversible white matter hyperintensities on FLAIR combined with normal DWI scans, which indicate vasogenic edema $(3,10)$. The edema reported in RPES cases is typically completely reversible. In certain cases, angiography may reveal a rosary bead-like appearance, representing diffuse cerebral vasodilation and vasoconstriction (3). The patient reported in the present study presented diffuse symmetric vasogenic edema in multiple regions, particularly the watershed zones of the parietal, occipital and frontal lobes. In addition, MRA suggested features of vasoconstriction or string-of-beads appearance, which are reflective of brain hypoperfusion $(3,10)$. Notably, cerebral venous vasodilation was also observed in the current patient, which has seldom been reported in the literature $(6,8)$. This abnormal venous vasodilation in addition to the arterial vasoconstriction were completely reversed following the aforementioned treatments, resulting in the patient's recovery.

The mechanism of RPES induced by eclampsia has yet to be fully elucidated. Increased cerebral vascular resistance and vasospasm may be observed in patients with preeclampsia or eclampsia (11). Thus, the generally accepted hypertension or hyperperfusion hypotheses regarding the mechanism of RPES development suggest that hypertension exceeds the autoregulation limits of the brain, which leads to cerebral autoregulatory vasoconstriction and subsequent brain edema (12). However, watershed hypoperfusion and reduced brain perfusion in the posterior brain region have also been proposed by previous studies $(13,14)$. At present, the intrinsic mechanism of RPES is considered to be an evolving systemic process with hypoperfusion/vasoconstriction and the development of brain toxicity, involving immune system activation, endothelial cell injury and inflammatory cytokine responses $(15,16)$. The aforementioned toxicity-associated hypoperfusion/vasoconstriction leads to capillary bed injury and vasogenic edema. In addition, hypertension in combination with eclampsia further reduces brain perfusion and induces ischemia. Thus, reducing the blood pressure of the patient can reduce vasoconstriction, improve perfusion, reverse watershed edema and subsequently improve clinical manifestations.
In conclusion, RPES associated with LPE is rare and may be easily misdiagnosed. The present case indicates the importance of considering the possibility of LPE without typical preeclampsia symptoms even several weeks after delivery. In addition, clinicians should be familiar with the artery constriction features of RPES on head radioimaging scans. Early diagnosis, adequate treatment and support are important for the patient's outcome.

\section{References}

1. Douglas KA and Redman CW: Eclampsia in the United Kingdom. BMJ 309: 1395-1400, 1994

2. Lubarsky SL, Barton JR, Friedman SA, Nasreddine S, Ramadan MK and Sibai BM: Late postpartum eclampsia revisited. Obstet Gynecol 83: 502-505, 1994.

3. Bartynski WS: Posterior reversible encephalopathy syndrome, part 1: Fundamental imaging and clinical features. AJNR Am J Neuroradiol 29: 1036-1042, 2008.

4. Postma IR, Slager S, Kremer HP, de Groot JC and Zeeman GG: Long-term consequences of the posterior reversible encephalopathy syndrome in eclampsia and preeclampsia: A review of the obstetric and nonobstetric literature. Obstet Gynecol Surv 69: 287-300, 2014.

5. Castrillo-Sanz A, Mendoza A, Gutiérrez-Ríos R, Zamora MI, Morollón N, Rodríguez-Sanz MF and Duarte J: Posterior reversible encephalopathy in a case of late-onset eclampsia. Rev Neurol 57: 112-116, 2013 (In Spanish).

6. Pezzi M, Le Piane E, Giglio AM, Pagnotta L, Scozzafava A, Tortorella V, Sergi A and Verre M: Posterior reversible encephalopathy syndrome in late postpartum eclampsia. Clin Ter 166: 68-71, 2015

7. Sibai BM: Diagnosis, prevention, and management of eclampsia. Obstet Gynecol 105: 402-410, 2005.

8. Minnerup J, Kleffner I, Wersching H, Zimmermann J, Schäbitz WR, Niederstadt T and Dziewas R: Late onset postpartum eclampsia: It is really never too late-a case of eclampsia 8 weeks after delivery. Stroke Res Treat 2010: pii. 798616, 2010.

9. Pizon AF and Wolfson AB: Postpartum focal neurologic deficits: Posterior leukoencephalopathy syndrome. J Emerg Med 29: 163-166, 2005.

10. Koch S, Rabinstein A,Falcone S and Forteza A: Diffusion-weighted imaging shows cytotoxic and vasogenic edema in eclampsia. AJNR Am J Neuroradiol 22: 1068-1070, 2001.

11. Qureshi AI, Frankel MR, Ottenlips JR and Stern BJ: Cerebral hemodynamics in preeclampsia and eclampsia. Arch Neurol 53: 1226-1231, 1996.

12. Strandgaard S, Olesen J,SkinhojE and Lassen NA: Autoregulation of brain circulation in severe arterial hypertension. Br Med J 1: 507-510, 1973.

13. Bartynski WS and Boardman JF: Catheter angiography, MR angiography and MR perfusion in posterior reversible encephalopathy syndrome. AJNR Am J Neuroradiol 29: 447-455, 2008.

14. Brubaker LM, Smith JK, Lee YZ, Lin W and Castillo M: Hemodynamic and permeability changes in posterior reversible encephalopathy syndrome measured by dynamic susceptibility perfusion-weighted MR imaging. AJNR Am J Neuroradiol 26: 825-830, 2005.

15. Bartynski WS: Posterior reversible encephalopathy syndrome, part 2: Controversies surrounding pathophysiology of vasogenic edema. AJNR Am J Neuroradiol 29: 1043-1049, 2008.

16. Kofler J, Bartynski WS, Reynolds TQ, Lieberman FS, Murdoch GH and Hamilton RL: Posterior reversible encephalopathy syndrome (PRES) with immune system activation, VEGF up-regulation and cerebral amyloid angiopathy. J Comput Assist Tomogr 35: 39-42, 2011. 